\title{
Ciliary and non-ciliary expression and function of PACRG during vertebrate development
}

\author{
Thomas Thumberger ${ }^{1,3}$, Cathrin Hagenlocher ${ }^{2}$, Matthias Tisler ${ }^{1}$, Tina Beyer ${ }^{1}$, Nina Tietze ${ }^{1}$, Axel Schweickert ${ }^{1}$,
} Kerstin Feistel ${ }^{2}$ and Martin Blum ${ }^{1 *}$

\begin{abstract}
Background: Park2-co-regulated gene (PACRG) is evolutionarily highly conserved from green algae to mammals. In Chlamydomonas and trypanosomes, the PACRG protein associates with flagella. Loss of PACRG results in shortened or absent flagella. In mouse the PACRG protein is required for spermatogenesis. The purpose of the present study was to analyze (1) the expression patterns of PACRG during vertebrate embryogenesis, and (2) whether the PACRG protein was required for left-right (LR) axis specification through cilia-driven leftward flow in Xenopus laevis.

Methods: PACRG CDNAs were cloned and expression was analyzed during early embryonic development of Xenopus, mouse, rabbit and zebrafish. Antisense morpholino oligonucleotide (MO) mediated gene knockdown was applied in Xenopus to investigate LR development at the level of tissue morphology, leftward flow and asymmetric marker gene expression, using timelapse videography, scanning electron microscopy (SEM) and whole-mount in situ hybridization. Results were statistically evaluated using Wilcoxon paired and $x^{2}$ tests.

Results: PACRG mRNA expression was found in cells and tissues harboring cilia throughout the vertebrates. Highly localized expression was also detected in the brain. During early development, PACRG was specifically localized to epithelia where leftward flow arises, that is, the gastrocoel roof plate (GRP) in Xenopus, the posterior notochord (PNC) in mammals and Kupffer's vesicle (KV) in zebrafish. Besides its association with ciliary axonemes, subcellular localization of PACRG protein was found around the nucleus and in a spotty pattern in the cytoplasm. A green fluorescent protein (GFP) fusion construct preferentially labeled cilia, rendering PACRG a versatile marker for live imaging. Loss-of-function in the frog resulted dose dependently in $L R$, neural tube closure and gastrulation defects, representing ciliary and non-ciliary functions of PACRG.
\end{abstract}

Conclusions: The PACRG protein is a novel essential factor of cilia in Xenopus.

Keywords: Cilia, Gastrulation defect, Left-right asymmetry, Leftward flow, Neural tube closure defect, PACRG, Park2, Xenopus

\section{Background}

PACRG was originally identified as a gene related to Parkinson's disease (PD) in humans [1,2]. In mammals PACRG shares a bidirectional promoter with Park2, the target gene for early onset juvenile PD. PACRG represents an evolutionarily very highly conserved gene, which is present from green algae to mammals $[1,3,4]$. Although a precise function has yet to be ascribed, the available evidence suggests that the PACRG protein is

\footnotetext{
* Correspondence: martin.blum@uni-hohenheim.de

'Institute of Zoology, Working group Embryology, University of Hohenheim, Garbenstraße 30, Stuttgart 70593, Germany

Full list of author information is available at the end of the article
}

associated with the ciliary axoneme: antibodies or green fluorescent protein (GFP) fusion proteins detected PACRG in flagellae of Chlamydomonas reinhardtii [3] and of Trypanosoma brucei [4] as well as in mouse spermatocytes [5]. Parallel RNAi-mediated knockdown of two paralogous genes in trypanosomes resulted in motility-impaired specimens with flagella of apparently normal length but outer microtubule doublet defects [4]. In the viable mutant mouse quaking $\left(q k^{\nu}\right)$ male fertility was lost due to a deletion of $P A C R G$, which resulted in failure to complete spermatogenesis [5]. Mutant mice were also affected by acquired hydrocephalus due to a defect in ependymal cilia function, resulting in reduced

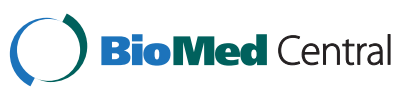


cerebrospinal fluid flow [6]. Structural investigations suggested that PACRG associated with nexin interdoublet links in trypanosomes [4]. In contrast, a localization between A and B tubules was proposed in the axoneme of Chlamydomonas flagellae [3]. Non-ciliary localizations were reported as well. PACRG was found in a large molecular chaperone complex containing heat shock proteins 70 and 90 as well as chaperonin components [2]. PACRG was further detected in Lewy bodies: these are neuronal inclusions frequently found in the brain of PD patients that are also positive for Parkin, the protein encoded by Park2 [2].

Cilia play a pivotal role during early vertebrate embryogenesis [7-10], with the establishment of the LR body axis as the first event where cilia are required [1114]. During gastrulation a ciliated epithelium forms at the posterior pole of the emerging notochord [15]. This epithelium harbors rotating monocilia, which due to their posterior polarization produce a leftward flow of extracellular fluid. Epithelia vary in shape and size but are structurally and functionally homologous [16]. They comprise the Kupffer's vesicle (KV) in bony fish, the gastrocoel roof plate (GRP) of amphibian embryos and the PNC in mammals. Experimental or genetic inhibition of flow, ablation or mispolarization of cilia or impairment of ciliary motility in all cases results in LR axis defects $[11,13,17,18]$. Downstream of leftward flow, the asymmetric Nodal gene cascade, consisting of the growth factor Nodal, its feedback inhibitor Lefty and the homeobox transcription factor Pitx2, is initiated in the left lateral plate mesoderm (LPM) and governs asymmetric organ morphogenesis and placement at later stages of development [7].

Here, we asked whether PACRG plays a role during embryogenesis as well, specifically during LR axis formation. PACRG expression was predominantly found in tissues harboring cilia in early frog, mouse, rabbit and zebrafish embryos. A GFP fusion protein labeled cilia in the frog GRP and epidermis. Gene knockdown in the frog demonstrated an embryonic role of PACRG in gastrulation, LR development and neural tube closure.

\section{Methods}

\section{Cloning of constructs}

Total RNA was isolated from embryos of various stages (frog, mouse, rabbit and zebrafish) and cDNAs were prepared using standard protocols. Primers for PCR amplification of $X$. laevis PACRG (accession number JQ771622) were designed based on $X$. tropicalis expressed sequence tags (accession numbers CX959700.1, CU025070.1): untranslated region (UTR) forward 5' ${ }^{\prime}$ TAGGCAACCGAAC GTAAACAACAG-3'; forward 5'-ATGGTGTTTGAGAC AAGCAAAGCAACA-3'; reverse 5'-GTTCAGCAAGCA GGATTCAT-3'. In order to clone the enhanced GFP
(eGFP) fusion construct, BamHI and XhoI restriction sites were introduced into forward and reverse primers, respectively. eGFP was cloned using primers forward $5^{\prime}$ 'CTCGAGATGGTGAGCAAGGGCGAGGAGC-3' (including XhoI site); reverse 5'-TCTAGATTACTTGTA CAGCTCGTCCATG-3' (including XbaI site).

Xenopus PACRG and eGFP were ligated into the BamHI/XbaI linearized CS2+ vector. The Xenopus rescue-eGFP construct was cloned using mutated PACRG forward primer $5^{\prime}$-ATGGTCTTCGAAACTAGTAAGG CAACA-3' to prevent morpholino oligonucleotide (MO) binding. Mouse PACRG (accession number BC120740.1) was cloned using primers forward 5'-CCCTCTCCT CCCCTAAACTC-3'; reverse 5'-GGTCAGTTCAGCAA GCACG-3'. A rabbit PACRG fragment was cloned using primers designed to match regions conserved between human (accession number BC044227.1) and mouse PACRG (see above): forward 5' - ATGCCGAAGAGGAC TAAACTGCTG-3'; reverse 5' ${ }^{\prime}$-ACCTACGAGTCTTGC TTGCT-3' (accession number JQ771623). Zebrafish PACRG (accession number ENSDARG00000004736) was cloned using primers forward 5' -ATGAGAACCTTTGAA CCTTTGGCTA-3'; reverse 5' -GTTGAGAAGGCAGGAC TCGTAGGTGGG-3'.

\section{RNA in situ hybridization, immunohistochemistry and histological analysis}

Embryos or explanted larval brains were fixed in MEMFA (1 part of (1M MOPS (pH 7.4, Roth), 20 mM EGTA (Applichem), and $10 \mathrm{mM} \mathrm{MgSO}$,(Applichem)), 8 parts $\mathrm{H}_{2} \mathrm{O}$ and 1 part formaldehyde (37\%, Roth)) or $4 \%$ paraformaldehyde (PFA, Roth) for $2 \mathrm{~h}$ and processed following standard protocols [18]. Digoxigenin-labeled (Roche) RNA probes were prepared from linearized plasmids using SP6 or T7 RNA polymerase (Promega). In situ hybridization was according to [19]. Immunohistochemistry was performed on whole-mount embryos fixed in $4 \%$ PFA for $1 \mathrm{~h}$ at room temperature. Embryos were processed according to standard procedures [18]. Antibodies used include mouse monoclonal antibody directed against acetylated alpha tubulin (1:700; Sigma), rabbit polyclonal antibody directed against PACRG (1:100, Rockland Immunochemicals, Inc.) and Cy2-conjugated or Cy3-conjugated secondary polyclonal rabbit or sheep anti mouse antibodies (Jackson Immunoresearch or Sigma; both 1:250). RNA encoding membrane red fluorescent protein (mRFP) (50 to $100 \mathrm{ng} / \mu \mathrm{l}$ ) or rhodamine-B dextran (0.5 to 1.0 $\mu \mathrm{g} / \mu \mathrm{l}$; Molecular Probes) were used as lineage tracers. For histological analysis embryos were embedded in gelatinalbumin and sectioned on a vibratome at $30 \mu \mathrm{m}$ (standard) to $40 \mu \mathrm{m}$ (brain sections). Statistical calculations of marker gene expression patterns were performed using Pearson's $\chi^{2}$ test (statistical R; http://cran.r-project.org/). SEM analysis was performed as described [20]. GRP cilia 
and cell parameters were determined in a square of $1,000 \times 1,000$ pixels (magnification 500-fold, corresponding to $86 \mu \mathrm{m}^{2}$ ) at the center of GRP in SEM pictures [21]. Cilia lengths, polarization (posterior, central, other) and cell surface areas were determined manually in ImageJ [22]. Ciliation rates were calculated as the ratio of cilia over cells (separately in each GRP SEM photograph). Posterior polarization was quantified for each GRP and statistical significances were calculated by Student's $t$ test in statistical R (http://cran.r-project.org/). The whiskers of the box plots extend to maximal $1.5 \times$ interquartile range (IQR), outliers are displayed as dots.

\section{Microinjections and MO-mediated knockdown of frog PACRG}

Embryos were injected at the four to eight cell stage using a Harvard Apparatus set-up. Drop size was calibrated to about 7-8 nl/injection. Morpholinos (Gene Tools, Philomath, OR, USA) were used at 0.4-2 pmol/ embryo as indicated. Lineage tracer RNAs were prepared using the Ambion message machine kit (Ambion) and diluted to a concentration of about 50-100 ng/ $\mu \mathrm{l}$. In all experiments care was taken to exclusively use four to eight cell embryos with a clear dorsoventral segregation of pigment $[23,24]$, and only correctly targeted specimens (controlled by coinjected lineage tracer) were processed for further analysis. The AUG blocking MO for frog PACRG (PACRG-MO) comprised 5'-TGCTTGT CTCAAACACCATATTCAC-3'.

\section{Video analysis of cilia, blastopore closure and leftward flow}

Fluorescent in vivo imaging of epidermal and GRP cilia was performed following injection of $80 \mathrm{ng} / \mu \mathrm{l} P A C R G$ eGFP mRNA. Timelapse sequences were recorded on a Zeiss Axioskop equipped with a CCD camera (AxioCam Hsm, Zeiss) using AxioVision 4.6 (Zeiss) at 62 fps (beating cilia) or $2 \mathrm{fps}$ (leftward flow). For blastopore closure timelapse acquisition, specimens were mounted onto an inverse microscope in a glass-bottom Petri dish onto a nitex mesh and cultivated in $0.1 \times \mathrm{MBSH}$ (Modified Barth's Saline). Timelapse movies were acquired at one frame every 2 minutes. Preparation of dorsal explants, recording of timelapse movies, processing and analysis of leftward flow were according to [18]. Significances were calculated by Student's t test in statistical R (http:// cran.r-project.org/). The whiskers of the box plot extend to maximal $1.5 \times \mathrm{IQR}$.

\section{Results and discussion}

Cloning and expression analysis of PACRG mRNA during vertebrate embryonic development

PACRG cDNAs were cloned from frog, mouse, rabbit and zebrafish. Alignment of deduced amino acid sequences

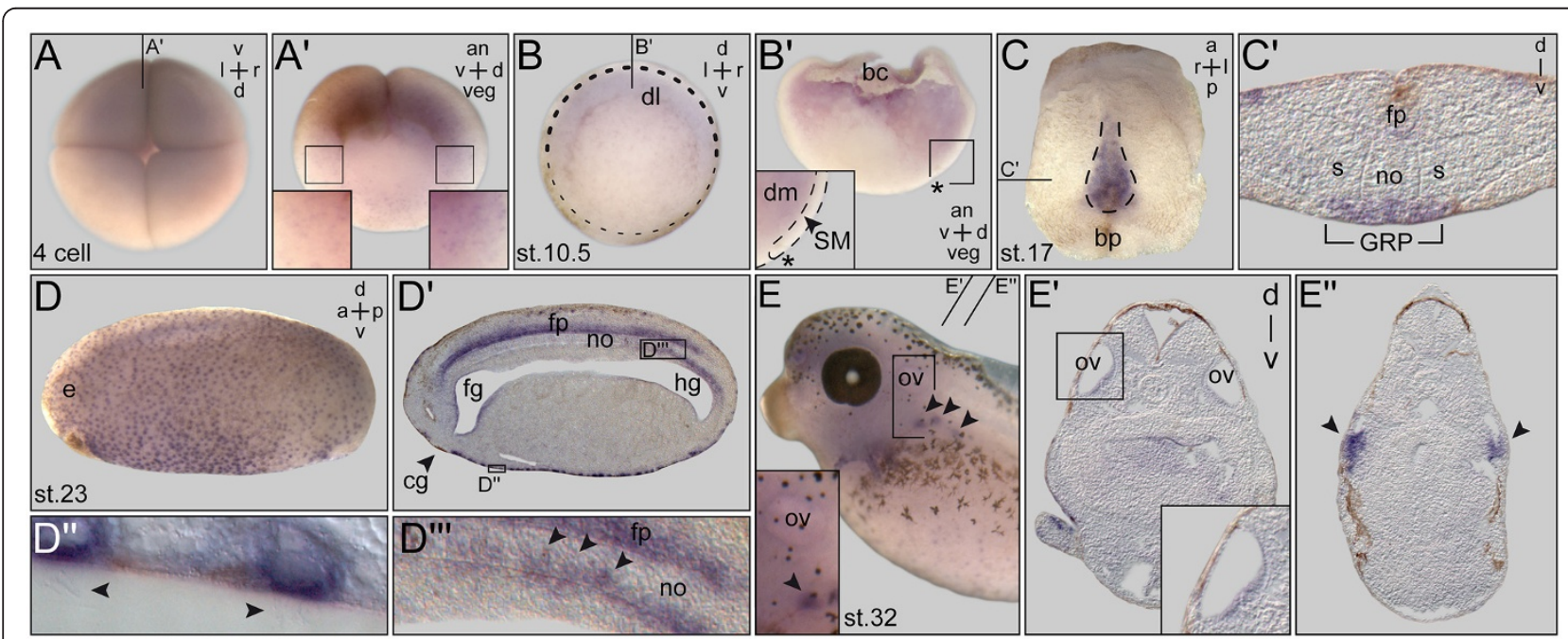

Figure 1 PACRG expression during early Xenopus development. (A) Expression at the four-cell stage (top view). (A') Sagittal hemisection of embryo shown in (A). Enlargements (boxes) indicate higher expression levels on the dorsal side. (B) Gastrula embryo. Persistent differences in staining intensities between dorsal and ventral side. (B') Sagittal hemisection (plane indicated in (B)) revealing mRNA localization in deep mesoderm. Note that the superficial mesoderm (SM) was free of PACRG mRNA (inset in ( $\left.B^{\prime}\right)$ ). Dorsal lip marked by asterisk. (C) Expression in the gastrocoel roof plate (GRP) at stage 17 (dorsal explant shown in ventral view). (C') Histological section (plane marked in (C)). (D) Expression at stage 23 in ciliated cells of the epidermis and the floor plate (sagittal section shown in ( $\left.\mathbf{D}^{\prime}\right)$ ). Enlargements show multiciliated skin cells ((D"); cilia indicated by arrowheads) and GRP cells after intercalation into the notochord ((D'"); arrowheads). (E) Staining in the otic vesicle and nephrostomes (arrowheads). ( $\left.\mathbf{E}^{\prime}, \mathbf{E}^{\prime \prime}\right)$ Histological sections (levels indicated in (E)) highlighting expression in the otic vesicle ( $\left.E^{\prime}\right)$ and nephrostomes

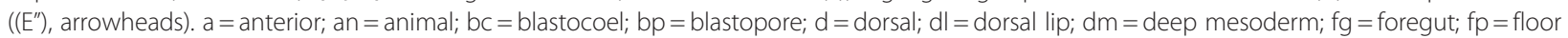
plate; $h g=$ hindgut; $I=$ left; $n o=$ notochord; ov = otic vesicle; $p=$ posterior; $r=$ right; $s=$ somite; $v=$ ventral; veg = vegetal. 
revealed high conservation between vertebrate species (Additional file 1: Figure S1). Expression patterns during development have so far not been described in vertebrates with the exception of zebrafish, where data from an in situ screen have been deposited into the Zfin database [25]. Embryonic expression was analyzed by whole mount in situ hybridization (WMISH) of staged embryos. In Xenopus, maternal mRNA was detected at the four-cell stage (Figure 1A). A punctate pattern was observed, which appeared slightly enriched on the dorsal compared to the ventral side, and more pronounced animally compared to the vegetal half (Figure 1A'). Zygotic expression was detected at the onset of gastrulation in the marginal zone, with again stronger signals on the dorsal side (Figure 1B). A hemisection revealed expression in the deep mesodermal layer (Figure 1B'). As cilia have not been reported during these early stages, expression indicated potential non-ciliary functions of $P A C R G$. The first cilia-related staining was found in the GRP from stage 13 onwards (Figure 1C,C' and data not shown). No signals were seen in the superficial mesoderm (SM), from which the GRP derives during gastrulation (Figure 1B'). Onset of PACRG expression thus correlated with the outgrowth of cilia, unlike other ciliary genes such as Foxj1 or dnah9, which are already induced in the SM and persist to be expressed in the GRP proper [18,26]. Multiciliated epidermal cells started to show PACRG mRNA localization at stage 17 (Figure 1C'), and maintained expression throughout embryogenesis (Figure 1D,E). Additional prominent sites of expression in ciliated cells were seen in the floor plate (Figure 1D'),
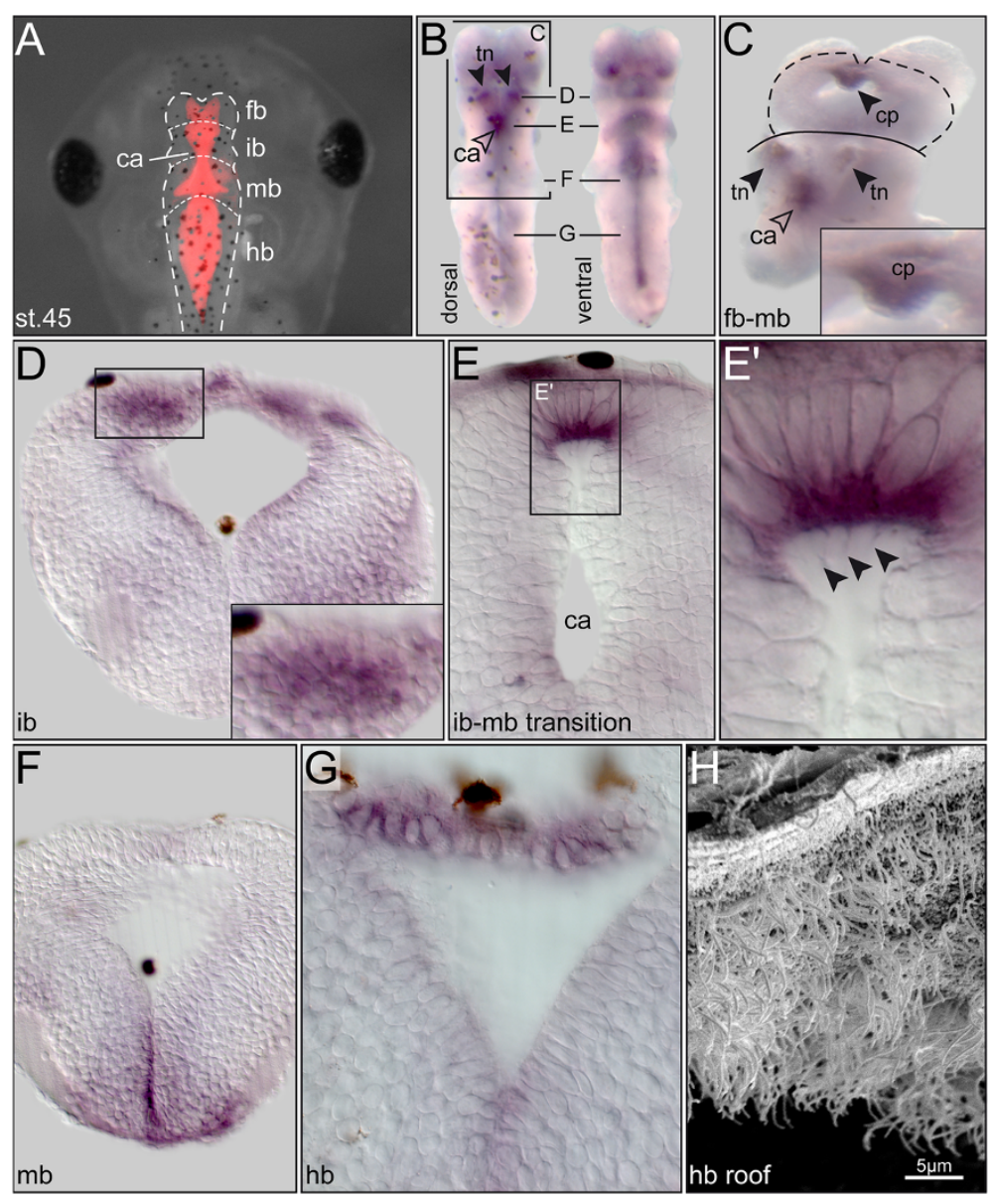

Figure 2 PACRG expression in the developing Xenopus brain. (A) Brain anatomy of the 3-day tadpole (stage 45), as highlighted by rhodamine dextran injection into the hindbrain ventricle. (B) Isolated brain following in situ hybridization with an antisense PACRG probe, shown in dorsal (left) and ventral (right) view. (C) Whole-mount of brain in which the hindbrain was removed and a dorsal cut was introduced between forebrain and interbrain to expose the choroid plexus (higher magnification shown in inset). (D-G) Transversal histological sections; levels indicated in (B), dorsal side up. (D) Staining in the thalamic nuclei. (E) Localized expression in roof of cerebral aqueduct (cilia highlighted by arrowheads in the enlargement shown in $\left(\mathbf{E}^{\prime}\right)$ ). (F) Expression in ventral midline of midbrain region. (G) Staining in roof of hindbrain. (H) Scanning electron micrograph, demonstrating multiciliated cells in roof of hindbrain. $\mathrm{ca}=$ cerebral aqueduct; $\mathrm{cp}=$ choroid plexus; $\mathrm{fb}=\mathrm{forebrain}$; $\mathrm{hb}=$ hindbrain; $\mathrm{ib}=$ interbrain; $\mathrm{mb}=$ midbrain; $\mathrm{tn}=$ thalamic nucleus. Note that dark brown spots in whole-mount brain specimens (B) and sections (D-G) represent melanocytes. 
nephrostomes (Figure 1E,E") and otic vesicle of the 2-day tadpole (Figure 1E,E'). Interestingly, notochordal GRP cells maintained PACRG staining at post-flow stages, when cells intercalated into the overlying notochord (Figure 1D"'; [27]).

Expression in the central nervous system was investigated in Xenopus whole-mount brain explants. The brain was interesting, because (1) adult $q k^{\nu /} q k^{\nu}$ mice develop hydrocephalus, a phenotype related to impaired cilia function [6], and (2) PACRG mRNA and protein was described to be expressed in regional brain areas of newborn and adult mice such as the lateral ventricles, the third and fourth ventricle, the aqueduct of Sylvius and the choroid plexus [28,29]. Brain samples were analyzed in 3-day (stage 40, data not shown) and 5-day (stage 45) tadpoles with comparable results (Figure 2). PACRG-specific signals were detected in multiciliated choroid plexus cells [28], thalamic nuclei and in the ventral midline (Figure 2A-C). Whether PACRG, in the cells of the ventral midline, has a ciliary or non-ciliary expression has not been determined so far. Interestingly, the specific expression in the thalamic nuclei (Figure 2D) correlates with a major site of non-dopaminergic neuron degeneration in the brain of PD patients [30]. Histological sections revealed staining in ciliated cells at the dorsal roof of the cerebral aqueduct (that is, the forming aqueduct of Sylvius; Figure 2E,E') and in the roof of the hindbrain (Figure 2G,H). In summary, expression patterns homologous to the one in the adult mouse brain were already visible during frog tadpole development, suggesting a function in the developing embryonic brain.

Rabbit, mouse and zebrafish embryos were investigated with a specific emphasis on the ciliated epithelia relevant for leftward flow. Figure 3 shows that PACRG mRNA was present in the PNC of rabbit and mouse as well as in the zebrafish KV. mRNA transcripts were localized to other ciliated tissues as well, such as the floor plate in all species and in the mouse otic vesicle. Comparable to Xenopus signals were also detected in the ventral midline of the developing mouse brain at E9.5 (Figure $3 C, C^{\prime}$ ). These data indicate that primary amino acid sequences and embryonic expression patterns were likewise conserved among the vertebrates. This notion is further supported by data available from the above-mentioned zebrafish expression screen. There, PACRG expression was additionally annotated in the otic vesicle, midbrain, pronephros, tegmentum and lateral line organ, all of which are ciliated structures [25]. Taken together, our expression analysis demonstrated a highly conserved pattern of PACRG mRNA localization in ciliated embryonic tissues, consistent with the axonemal localization reported in Chlamydomonas and trypanosomes $[3,4]$. In addition, signals were found in

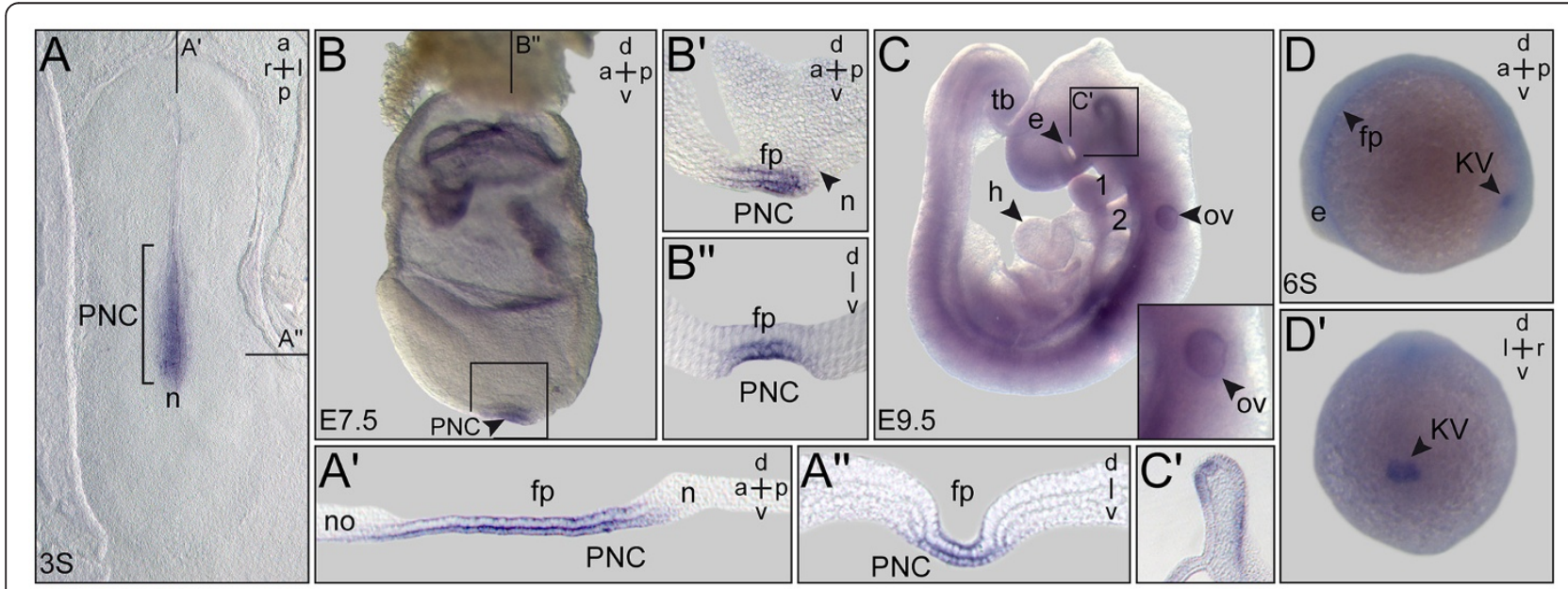

Figure 3 PACRG expression during early rabbit, mouse and zebrafish development. Whole-mount in situ hybridization of staged embryos. (A) Rabbit. Three somite (3S) stage blastodisc revealing PACRG expression in the posterior notochord (PNC) and floor plate. (A') Sagittal section. $\left(\mathbf{A}^{\prime \prime}\right)$ Transversal section (level marked in (A)). Note that the sagittal section revealed absence of PACRG from the node (A'). (B,C) Mouse. (B) E7.5

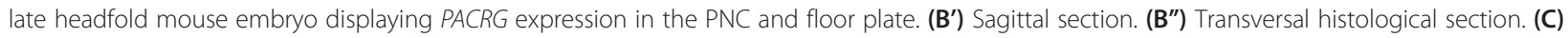
E9.5 embryo. Otic vesicle shown in higher magnification in inset. Box indicates brain region of which a histological section is provided in $\left(\mathbf{C}^{\prime}\right)$. ( $\left.\mathbf{C}^{\prime}\right)$ Histological sagittal section, revealing staining in ventral midline of the brain. (D) 6-Somite stage zebrafish embryo. PACRG expression in the Kupffer's vesicle (KV) and floor plate. $a=$ anterior; $d=$ dorsal; $e=e y e ; f p=$ floor plate; $h=$ heart; $I=l e f t ; n=$ node; no = notochord; ov =otic vesicle; $\mathrm{p}=$ posterior; $\mathrm{r}=$ right; $\mathrm{s}=$ somite; $\mathrm{tb}=$ tail bud; $\mathrm{v}=$ ventral. 
non-ciliated cells during early cleavage stages and in brain regions not analyzed for the presence of cilia as yet.

\section{PACRG protein localizes to cilia and intracellular compartments}

Further analyses of PACRG were performed in Xenopus embryos, as this model organism is ideally suited to study LR asymmetry [31]. To confirm axonemal protein localization, immunohistochemistry was applied in which axonemal microtubules and PACRG were stained simultaneously. Figure 4A demonstrates PACRG localization along monocilia of GRP cells at stage 17. Additional PACRG signals were detected in a punctate pattern in the cytoplasm and at the plasma membrane (Figure 4A'), indicating axonemal as well as cytoplasmic functions. Localization to multiciliated skin cells of stage 25 tadpoles is depicted in Figure 4B. Interestingly, PACRG was again found within the cell as well. Besides scattered punctae a concentration was seen around the nucleus (Figure 4D), which was not further characterized. PACRG localization to cytoplasmic vesicles and to the perinuclear area have previously been reported in cultured primary neurons from the mouse midbrain [2], supporting the specificity of the observed patterns. As cilia in the GRP, nephrostomes, otic vesicle, brain and on epidermal cells are all motile, we wondered whether PACRG localized to primary immotile cilia as well. Primary cilia have been described in the pronephric duct of Xenopus tadpoles [32]. We therefore analyzed PACRG expression by immunohistochemistry at stage 37/38. As shown in Additional file 2: Figure S2, a faint though clear signal was detected along the axoneme of primary cilia in the pronephric duct. In summary, PACRG was found to localize to both motile and immotile cilia.

In order to confirm the immunohistochemistry data, we injected a PACRG-eGFP fusion construct into fourcell Xenopus embryos and targeted the mRNA to the GRP or epidermal cells. As shown in Figure 4E,F, the fusion protein marked both monocilia on the GRP and cilia of multiciliated skin cells. Vesicle-like structures and the perinuclear region were positive for the fusion
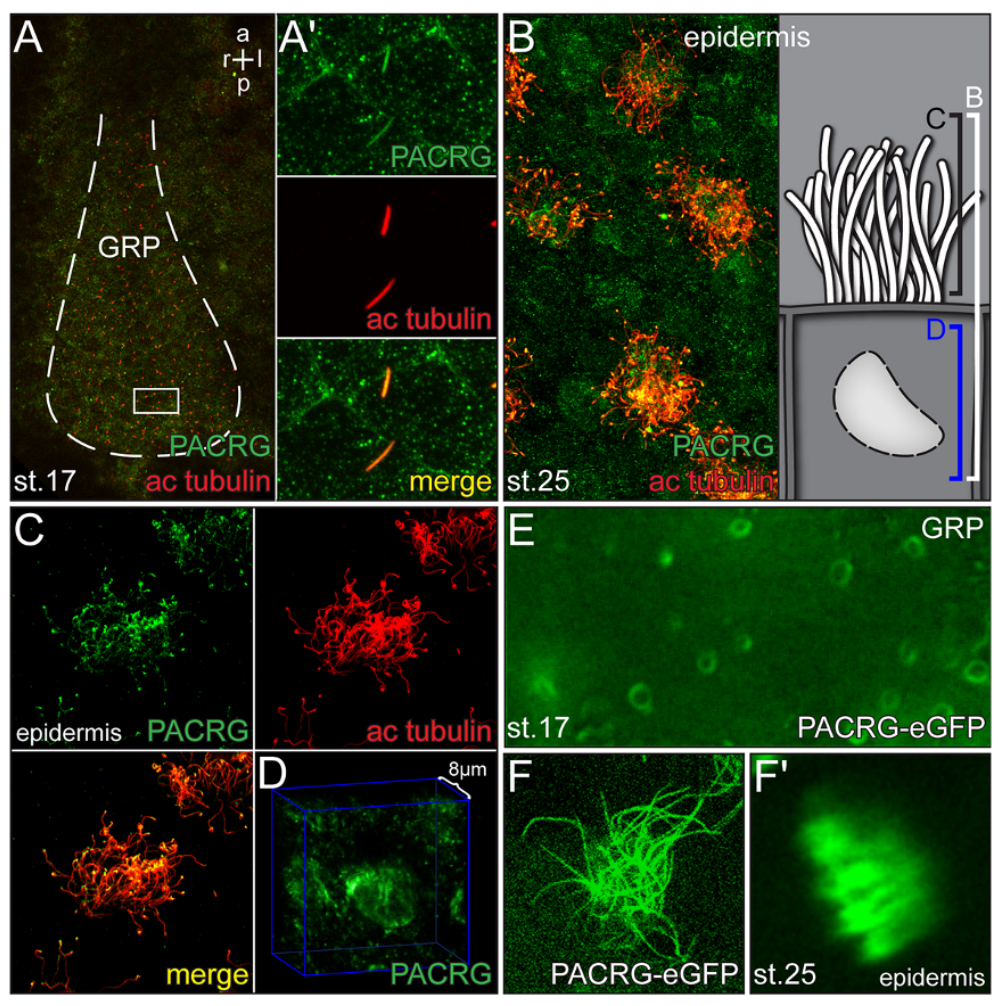

Figure 4 PACRG protein localization. (A-D) Immunohistochemistry. PACRG shown in green and acetylated tubulin in red. (A) Ventral view of gastrocoel roof plate (GRP) (dashed white line) at stage 17. (A') Enlargement of GRP cilia. (B) Epidermis. Overview and schematic depiction of multiciliated epidermal cell. (C,D) Enlargements of cilia (C) and cytoplasmic PACRG staining (D). Note that within the cell PACRG localizes to vesicle-like structures and the perinuclear region. (E,F) Expression of a PACRG-enhanced green fluorescent protein (PACRG-eGFP) fusion protein. (E) Maximum intensity projection of timelapse movie of stage $17 \mathrm{GRP}$ cilia, demonstrating elliptic shape of ciliary beating. (F) Squash preparation and $\left(\mathbf{F}^{\prime}\right)$ maximum intensity projection of timelapse movie of stage 25 multiciliated skin cell. $a=$ anterior; $a c=$ acetylated; $\mid=$ left; $p=$ posterior; $r=$ right. 
protein as well (not shown), demonstrating that the fusion protein localized in an identical manner as the endogenous PACRG.

The ciliary localization of PACRG-eGFP afforded the opportunity of testing whether this fusion protein enabled live imaging of motile cilia in the frog Xenopus. The thickness of dorsal explants and the high yolk content of cells resulted in scattering of polarized light, which prevented in vivo imaging of GRP cilia in top view in the past [13]. Additional file 3, movie 1 shows a field of GRP cilia, confirming their rotational and, due to the posterior tilt, elliptical beat pattern (see also Figure 4E). The whip-like wave form of epidermal cilia bundles could likewise be recorded (Additional file 3: Movie 1). These data demonstrated that PACRG-eGFP could be used as a cilia marker for live imaging in frog, and perhaps in other vertebrate model organisms as well.

\section{LR axis defects in PACRG morphants}

In order to investigate the function of $P A C R G$ during Xenopus LR development, an antisense morpholino oligonucleotide (MO) was designed which targeted the translational start site. PACRG-MO or a random control MO (co-MO) were injected into the GRP lineage by targeting the dorsal marginal zone at the four-cell stage as described [18]. Embryos were cultured until control uninjected specimens reached stage 34 . Dose-dependently a series of axial defects were recorded (see below). Following injections of $0.4 \mathrm{pmol} P A C R G-\mathrm{MO}$ per embryo specimens developed with wild-type dorsoanterior index (DAI; [33]) of $5(n=89 / 91)$. Alterations of dorsoanterior development $(\mathrm{DAI} \neq 5)$ frequently indicate midline defects, which inevitably cause altered LR marker gene expression and organ situs [34,35]. Therefore LR parameters were only evaluated in DAI5 PACRG morphants.

In a first set of experiments we asked whether PACRG was required for LR development. To assess the induction of the asymmetric Nodal cascade in the left LPM expression of Pitx2c in 2-day tadpoles was analyzed following PACRG knockdown. As shown in Figure 5A Pitx2c was predominantly absent in the left LPM of morphant specimens in a dose-dependent manner. Next

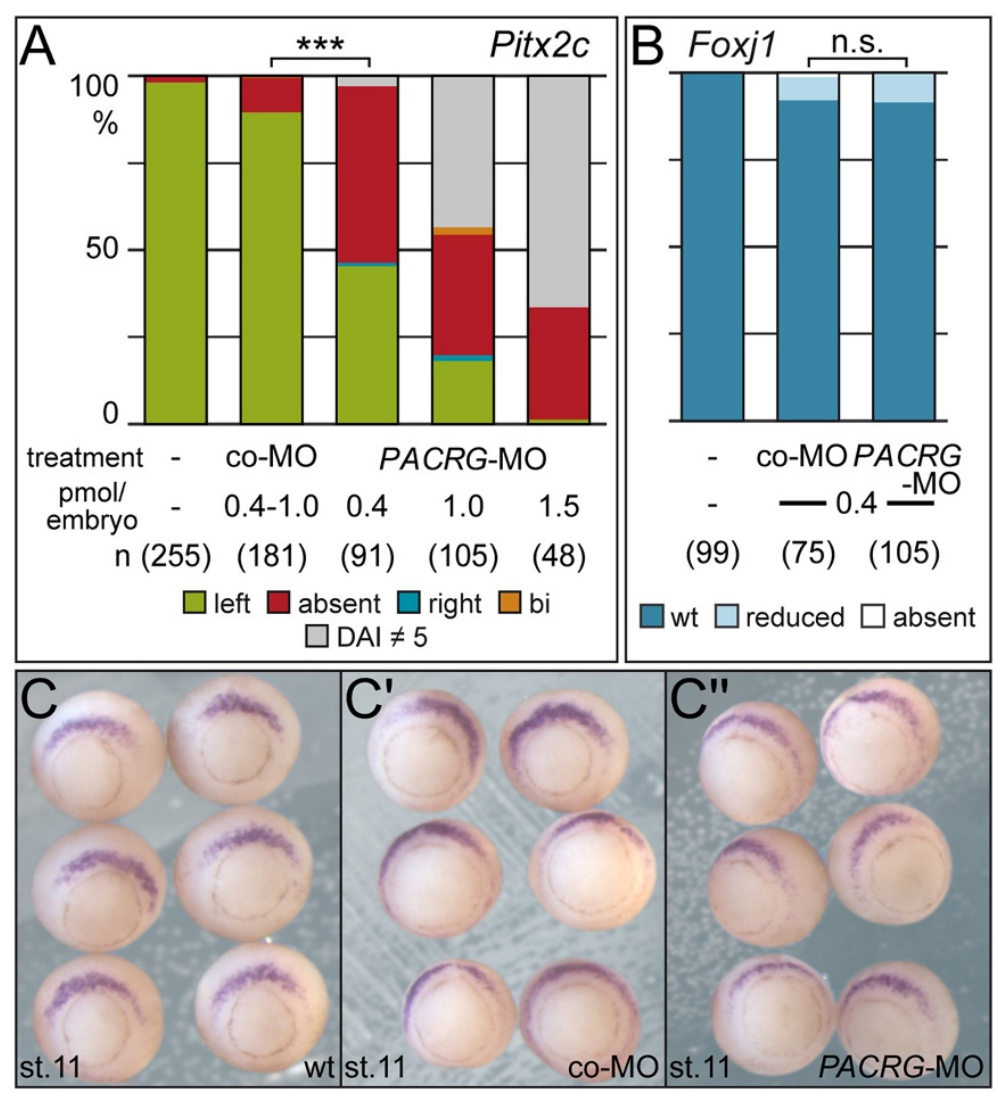

Figure 5 Impaired laterality in PACRG morphants in the absence of superficial mesoderm (SM) defects. (A) Pitx2c gene expression in the lateral plate mesoderm (LPM). Note that the morpholino oligonucleotide (MO) dose chosen (0.4 pmol/embryo) did not affect dorsoanterior development. (B,C) Unaltered Foxj1 expression levels in the SM of morphants. Assessment of expression levels was as described previously [36]. Embryos in $(C)$ are shown in vegetal view, dorsal side up. Numbers in brackets represent number of analyzed specimens. ${ }^{* * *}=$ very highly significant $(P<0.001)$; n.S., not significant $(P=0.891)$. 
we wondered whether the SM was specified normally, as SM defects result in aberrant laterality as well [21,31]. To that end expression of Foxj1, the master control gene of motile cilia [26] was assessed at stage 11. Figure 5BC" demonstrates that Foxj1 was not affected by knockdown of PACRG, indicating that the SM was specified correctly and PACRG should be required during flow stages.

To analyze leftward flow, dorsal explants of co-MO and PACRG-MO injected embryos were prepared, fluorescent beads were added and timelapse videos were recorded and evaluated as described [17,18]. Additional file 4: Movie 2 and Figure $6 \mathrm{~A}-\mathrm{C}$ show that very few directed beads were detectable in PACRG morphants, and quality of flow was severely affected $(P<0.001)$. As a measure of flow quality, the dimensionless number rho was used, which represents the mean resultant length of all bead trajectory angles [18], with a rho value of 1 representing beads moving in one direction and rho $=0$ representing randomness of bead trajectories. As impaired flow frequently correlates with altered GRP morphology, ciliation and/or cilia function $[17,18,21]$, dorsal explants were examined by SEM. The outline of the GRP was deformed in morphants as compared to wildtype specimens (Figure 6D,E). The mean ciliation rate dropped significantly, and mean cilia lengths and rate of posterior polarization of residual cilia were reduced as well (Figure 6F). In addition the mean apical surface area of morphant GRP cells was enlarged (Figure 6F). Taken together, these results demonstrate that PACRG was required for GRP morphology, ciliation, leftward flow and thus left-asymmetric initiation of the nodal signaling cascade in the frog Xenopus. The lack of a like phenotype in mouse, where LR defects were not recorded in the $q k^{v}$ mutant which harbors a PACRG deletion, might be due to functional redundancy, as a PACRG-related protein has been annotated in the database (NM_025755.3; [37]). A second PACRG gene is not a

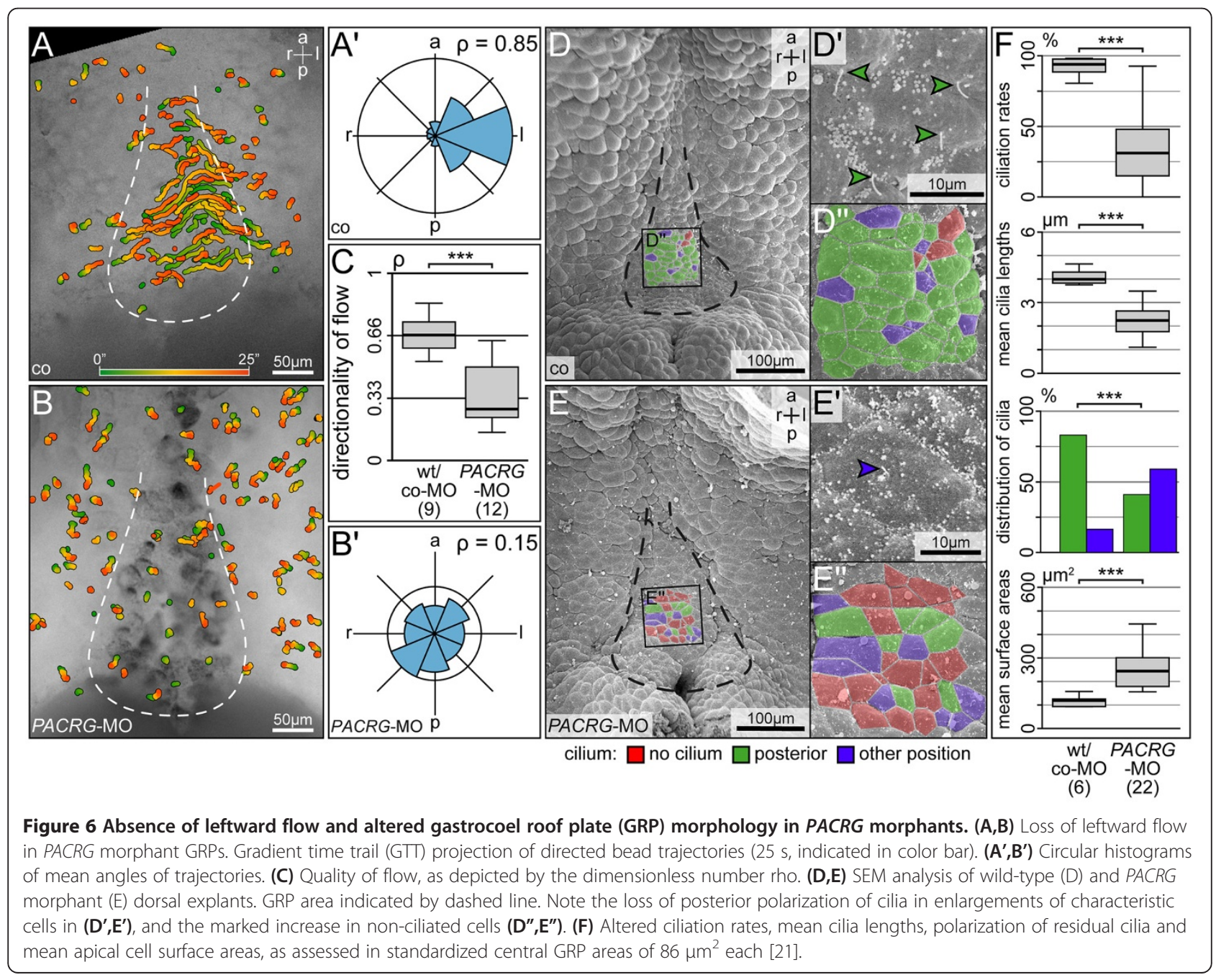


unique feature of mammals, as trypanosomes have two genes as well [4]. PARK2 co-regulated-like has been annotated in Xenopus as well (http://www.xenbase.org), however, expression and function have not been assessed so far. Sequences differ such that our MO would not have targeted translation of PACRG-l. It remains to be seen if and to what extend PACRG and PACRG-l complement each other.

\section{Gastrulation and neural tube closure defects in morphants}

At $\mathrm{MO}$ concentrations $>0.4 \mathrm{pmol} /$ embryo a series of phenotypes was encountered, ranging from mild neural tube closure defects to microcephaly or complete loss of cranial structures (Figure 7A). At doses $\geq 2 \mathrm{pmol} / \mathrm{embryo}$, severe gastrulation defects occurred, with a failure of blastopore closure on the injected side. In order to follow the appearance of high dose phenotypes during development, timelapse movies were recorded. Additional file 5: Movie 3 and single frames taken from the onset of dorsal lip formation through blastopore closure demonstrate that the dorsal lip did not form in morphants as compared to control embryos (Figure 7B,C). Notably, the ventral lip, which was not targeted by the $\mathrm{MO}$, appeared in time (Figure $7 \mathrm{C}^{\prime}$ ). Dorsal lip formation requires apical constriction in order to give rise to bottle cells [38]. Interestingly, apical constriction of bottle cells was shown to be dependent on stable microtubules [38,39]. PACRG binds to and bundles microtubules in vitro [40], suggesting that PACRG was required for microtubule function in bottle cell apical constriction. The expression pattern of acetylated alpha tubulin in the deep mesoderm of the marginal zone (reflecting stable microtubules), which colocalizes to PACRG mRNA (see also Figure 1B', and Figure 7D'), supports this notion.

Neural tube closure depends on apical constriction of neural plate cells as well, and as with bottle cell formation

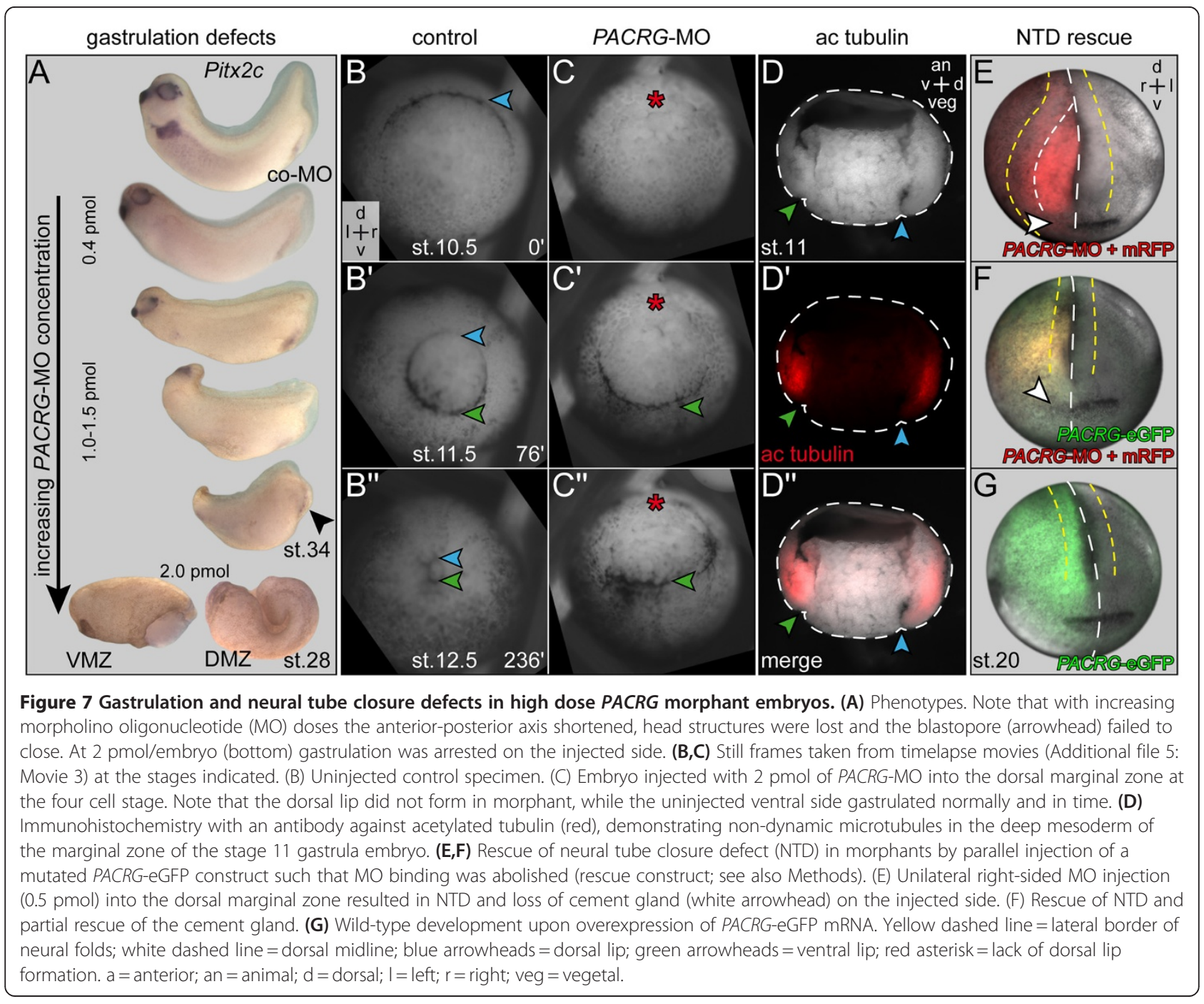


this process has been shown to require intact microtubules [41]. Neural tube closure defects (NTD) seen at intermediate PACRG-MO doses might therefore be related to altered assembly of parallel microtubule arrays as well. NTD represented a specific PACRG phenotype, as we were able to rescue closure by coinjection of PACRGeGFP mRNA using a construct in which the MO binding site was mutated (Figure 7E-G). In summary, intermediate and high doses of MO caused phenotypes unrelated to cilia but associated with intracellular arrangement of ordered microtubule bundles.

\section{Conclusions}

Our study of PACRG in four vertebrate model organisms revealed a pronounced degree of conservation at the level of amino acid sequences and embryonic expression. In particular, $P A C R G$ was highly correlated with motile cilia during development, an aspect that we confirmed in depth by our functional analysis of LR development in PACRG morphants in Xenopus. The remarkable nonciliary PACRG functions are worth being analyzed in greater depth in future studies. The preliminary evidence presented here points to a more general role related to non-dynamic microtubules in their recently shown involvement in apical cell constriction. In addition, the expression in the embryonic brain at sites where lesions in populations of non-dopaminergic neurons occur in PD patients may deserve further attention. Lastly, PACRG may serve as a versatile marker of motile cilia in live imaging.

\section{Additional files}

\section{Additional file 1: Additional Figure 1 High conservation of PACRG} amino acid sequences from zebrafish, human, rabbit, mouse and Xenopus laevis. Alignment of amino acid sequences derived from zebrafish (ENSDARG00000004736), human (BC044227.1), rabbit (JQ771623) mouse (BC120740.1) and Xenopus laevis (JQ771622) CDNAs. Variations were restricted to the $\mathrm{N}$-terminal part, encoded mostly in exon 1. Note that no protein domains of known function have as yet been ascribed to PACRG. Amino acids derived from rabbit primers used for PCR amplification are indicated with blue rectangles (see also Methods).

Additional file 2: Additional Figure 2 Localization of PACRG to primary cilia of the pronephric duct. Tadpoles at stage $37 / 38$ were fixed and processed for immunohistochemistry with antibodies specific for PACRG (green) and acetylated tubulin (red). Specimens were sectioned on a vibratome (30 $\mathrm{mm})$ and viewed in the confocal laser scanning microscope. The lumen of the pronephric duct is outlined by a white dashed line. White rectangles indicate regions shown in higher magnification in the lower left corner

Additional file 3: Movie 1 A PACRG-eGFP fusion construct labels monociliated and multiciliated cells in vivo. GRP cilia (stage 17; left) and multiciliated skin cells (stage 25; right) were labeled by injection of PACRG-eGFP at the four-cell stage. The first frame shows maximum intensity projection of timelapse movies, which play in real time. Note that cilia were imaged in top-down view, which in Xenopus due to the high yolk content of cells is not possible without labeling of cilia.

Additional file 4: Movie 2 Absence of leftward flow in PACRG morphants. Timelapse sequences of dorsal explant cultures to which fluorescent beads were added. Specimens were mounted dorsal side down (anterior to the top) and are viewed from the ventral side. Movie runs at $40 \times$ real time. Opening frame indicates orientation of GRP (white dashed lines). Videos were processed to yield gradient time trails (GTTs), that is, color-coded tracks of beads which reveal direction of transport and velocity of particles (from green to red; 25 ; [ 18]). Note that robust leftward flow of control specimen (left) was absent in morphant (right).

Additional file 5: Movie 3 Gastrulation defects in PACRG morphants. Timelapse sequences of control (left) and PACRG morphant embryo (right) from stage 9-12.5. PACRG-MO was injected into the prospective dorsal marginal zone at the 4-cell stage. Movies were recorded at 0.5 frames per minute and pause at stages 10 and 11 to demonstrate absence (red asterisk) of dorsal lip (blue arrowhead) formation and gastrulation via the ventral lip (green arrowhead) in morphant sample. Note that the unmanipulated ventral lip formed at the same time as in the uninjected control embryo. Embryos are shown in vegetal view, dorsal side up.

\section{Competing interests}

The authors declare that they have no competing interests.

\section{Acknowledgements}

We would like to thank Ray Keller, in whose lab the blastopore closure timelapse videos were recorded with the help of Dave Shook, Philipp Vick for providing the gastrula-stage tubulin immunohistochemistry and for critical reading of the manuscript, Susanne Bogusch for expert help with some of the experiments and Jochen Wittbrodt, in whose lab TT performed the zebrafish experiments. TT and TB were recipients of a PhD fellowship from the Landesgraduiertenförderung Baden-Württemberg, $\mathrm{CH}$ and KF are indebted to the Baden-Württemberg Stiftung for the financial support of their research by the Eliteprogramme for Postdocs; KF was supported by a Margarete-von-Wrangell fellowship, funded by the European Social Fund in Baden-Württemberg, and work in the Blum lab was funded through DFG grants BI285/9-2 and BI285/10-1.

\section{Author details}

${ }^{1}$ Institute of Zoology, Working group Embryology, University of Hohenheim, Garbenstraße 30, Stuttgart 70593, Germany. ${ }^{2}$ Institute of Zoology, Working group Neural Stem Cells, University of Hohenheim, Garbenstraße 30, Stuttgart 70593, Germany. ${ }^{3}$ Present address: Centre for Organismal Studies (COS), University of Heidelberg, Im Neuenheimer Feld 230, Heidelberg 69120, Germany.

\section{Authors' contributions}

TT carried out live imaging, PACRG mRNA and protein expression analysis in Xenopus and zebrafish, most of the functional experiments, prepared the figures and movies and helped to draft the manuscript; $\mathrm{CH}$ performed the expression and SEM analyses in the brain; MT participated in the morphant analysis; TB conducted the SEM analysis; NT cloned and analyzed PACRG expression in rabbit and mouse; KF analyzed the brain expression patterns and ventricular ciliation; AS contributed to the interpretation of the experimental data; MB supervised the study, interpreted the data and wrote the manuscript. All authors read and approved the final manuscript.

Received: 16 March 2012 Accepted: 30 May 2012

Published: 1 August 2012

\section{References}

1. West $A B$, Lockhart PJ, O'Farell C, Farrer MJ: Identification of a novel gene linked to parkin via a bi-directional promoter. J Mol Biol 2003, 326:11-19.

2. Imai Y, Soda M, Murakami T, Shoji M, Abe K, Takahashi R: A product of the human gene adjacent to parkin is a component of Lewy bodies and suppresses Pael receptor-induced cell death. J Biol Chem 2003, 278:51901-51910.

3. Ikeda K, Ikeda T, Morikawa K, Kamiya R: Axonemal localization of Chlamydomonas PACRG, a homologue of the human Parkin-coregulated gene product. Cell Motil Cytoskeleton 2007, 64:814-821.

4. Dawe HR, Farr H, Portman N, Shaw MK, Gull K: The Parkin co-regulated gene product, PACRG, is an evolutionarily conserved axonemal protein 
that functions in outer-doublet microtubule morphogenesis. J Cell Sci 2005, 118:5421-5430.

5. Lorenzetti D, Bishop CE, Justice MJ: Deletion of the Parkin coregulated gene causes male sterility in the quaking(viable) mouse mutant. Proc Natl Acad Sci USA 2004, 101:8402-8407.

6. Wilson GR, Wang HX, Egan GF, Robinson PJ, Delatycki MB, O'Bryan MK Lockhart PJ: Deletion of the Parkin co-regulated gene causes defects in ependymal ciliary motility and hydrocephalus in the quakingviable mutant mouse. Hum Mol Genet 2010, 19:1593-1602.

7. Hamada H, Meno C, Watanabe D, Saijoh Y: Establishment of vertebrate left-right asymmetry. Nat Rev Genet 2002, 3:103-113.

8. Hirokawa N, Tanaka Y, Okada Y, Takeda S: Nodal flow and the generation of left-right asymmetry. Cell 2006, 125:33-45.

9. Park TJ, Haigo SL, Wallingford JB: Ciliogenesis defects in embryos lacking inturned or fuzzy function are associated with failure of planar cell polarity and Hedgehog signaling. Nat Genet 2006, 38:303-311.

10. Marshall WF, Kintner C: Cilia orientation and the fluid mechanics of development. Curr Opin Cell Biol 2008, 20:48-52.

11. Nonaka S, Tanaka Y, Okada Y, Takeda S, Harada A, Kanai Y, Kido M, Hirokawa N: Randomization of left-right asymmetry due to loss of nodal cilia generating leftward flow of extraembryonic fluid in mice lacking KIF3B motor protein. Cell 1998, 95:829-837.

12. Okada Y, Takeda S, Tanaka Y, Belmonte J-Cl, Hirokawa N: Mechanism of nodal flow: a conserved symmetry breaking event in left-right axis determination. Cell 2005, 121:633-644.

13. Schweickert A, Weber T, Beyer T, Vick P, Bogusch S, Feistel K, Blum M: Ciliadriven leftward flow determines laterality in Xenopus. Curr Biol 2007, 17:60-66.

14. Essner JJ, Amack JD, Nyholm MK, Harris EB, Yost HJ: Kupffer's vesicle is a ciliated organ of asymmetry in the zebrafish embryo that initiates leftright development of the brain, heart and gut. Development 2005, 132:1247-1260.

15. Blum M, Andre P, Muders K, Schweickert A, Fischer A, Bitzer E, Bogusch S, Beyer T, van Straaten HWM, Viebahn C: Ciliation and gene expression distinguish between node and posterior notochord in the mammalian embryo. Differentiation 2007, 75:133-146.

16. Blum M, Weber T, Beyer T, Vick P: Evolution of leftward flow. Semin Cell Dev Biol 2009, 20:464-471.

17. Maisonneuve C, Guilleret I, Vick P, Weber T, Andre P, Beyer T, Blum M, Constam DB: Bicaudal $C$, a novel regulator of Dvl signaling abutting RNAprocessing bodies, controls cilia orientation and leftward flow. Development 2009, 136:3019-3030.

18. Vick P, Schweickert A, Weber T, Eberhardt M, Mencl S, Shcherbakov D, Beyer T, Blum M: Flow on the right side of the gastrocoel roof plate is dispensable for symmetry breakage in the frog Xenopus laevis. Dev Biol 2009, 331:281291

19. Belo JA, Bouwmeester T, Leyns L, Kertesz N, Gallo M, Follettie M, De Robertis EM: Cerberus-like is a secreted factor with neutralizing activity expressed in the anterior primitive endoderm of the mouse gastrula. Mech Dev 1997, 68:45-57.

20. Sulik K, Dehart DB, langaki T, Carson JL, Vrablic T, Gesteland K, Schoenwolf GC: Morphogenesis of the murine node and notochordal plate. Dev Dyn 1994, 201:260-278.

21. Beyer T, Danilchik M, Thumberger T, Vick P, Tisler M, Schneider I, Bogusch S, Andre P, Ulmer B, Walentek P, Niesler B, Blum M, Schweickert A: Serotonin signaling is required for wnt-dependent GRP specification and leftward flow in xenopus. Curr Biol 2012, 22:33-39.

22. Sbalzarini IF, Koumoutsakos P: Feature point tracking and trajectory analysis for video imaging in cell biology. J Struct Biol 2005, 151:182-195.

23. Klein SL: The first cleavage furrow demarcates the dorsal-ventral axis in Xenopus embryos. Dev Biol 1987, 120:299-304.

24. Danilchik MV, Black SD: The first cleavage plane and the embryonic axis are determined by separate mechanisms in Xenopus laevis. I. Independence in undisturbed embryos. Dev Biol 1988, 128:58-64.

25. Thisse B, Thisse C: Fast release clones: a high throughput expression analysis, ZFIN direct data submission.; 2004. http://zfin.org/cgi-bin/webdriver? Mlval=aa-pubview2.apg\&OID=ZDB-PUB-040907-1

26. Stubbs JL, Oishi I, Izpisúa-Belmonte JC, Kintner C: The forkhead protein Foxj1 specifies node-like cilia in Xenopus and zebrafish embryos. Nat Genet 2008, 40:1454-1460.
27. Shook DR, Majer C, Keller R: Pattern and morphogenesis of presumptive superficial mesoderm in two closely related species, Xenopus laevis and Xenopus tropicalis. Dev Biol 2004, 270:163-185.

28. Wilson GR, Tan JT, Brody KM, Taylor JM, Delatycki MB, Lockhart PJ: Expression and localization of the Parkin co-regulated gene in mouse CNS suggests a role in ependymal cilia function. Neurosci Lett 2009, 460:97-101.

29. Brody KM, Taylor JM, Wilson GR, Delatycki MB, Lockhart PJ: Regional and cellular localisation of Parkin co-regulated gene in developing and adult mouse brain. Brain Res 2008, 1201:177-186.

30. Bacci J-J, Kachidian P, Kerkerian-Le Goff L, Salin P: Intralaminar thalamic nuclei lesions: widespread impact on dopamine denervation-mediated cellular defects in the rat basal ganglia. J Neuropathol Exp Neurol 2004, 63:20-31.

31. Blum M, Beyer T, Weber T, Vick P, Andre P, Bitzer E, Schweickert A: Xenopus, an ideal model system to study vertebrate left-right asymmetry. Dev Dyn 2009, 238:1215-1225.

32. Tran U, Pickney LM, Ozpolat BD, Wessely O: Xenopus Bicaudal-C is required for the differentiation of the amphibian pronephros. Dev Biol 2007, 307:152-164.

33. Kao KR, Elinson RP: The entire mesodermal mantle behaves as Spemann's organizer in dorsoanterior enhanced Xenopus laevis embryos. Dev Biol 1988, 127:64-77.

34. Hamada $\mathrm{H}$ : Breakthroughs and future challenges in left-right patterning. Dev Growth Differ 2008, 50(Suppl 1):S71-S78.

35. Lee JD, Anderson KV: Morphogenesis of the node and notochord: the cellular basis for the establishment and maintenance of left-right asymmetry in the mouse. Dev Dyn 2008, 237:3464-3476.

36. Walentek P, Beyer T, Thumberger T, Schweickert A, Blum M: ATP4a is required for wnt-dependent foxj 1 expression and leftward flow in xenopus left-right development. Cell Reports 2012, 1:516-527.

37. Diez-Roux G, Banfi S, Sultan M, Geffers L, Anand S, Rozado D, Magen A, Canidio E, Pagani M, Peluso I, Lin-Marq N, Koch M, Bilio M, Cantiello I, Verde R, De Masi C, Bianchi SA, Cicchini J, Perroud E, Mehmeti S, Dagand E, Schrinner S, Nürnberger A, Schmidt K, Metz K, Zwingmann C, Brieske N, Springer C, Hernandez AM, Herzog S, et al: A high-resolution anatomical atlas of the transcriptome in the mouse embryo. PLoS Biol 2011, 9:e1000582.

38. Lee J-Y, Harland RM: Actomyosin contractility and microtubules drive apical constriction in Xenopus bottle cells. Dev Biol 2007, 311:40-52.

39. Lee J-Y, Harland RM: Endocytosis is required for efficient apical constriction during Xenopus gastrulation. Curr Biol 2010, 20:253-258.

40. Ikeda T: Parkin-co-regulated gene (PACRG) product interacts with tubulin and microtubules. FEBS Lett 2008, 582:1413-1418.

41. Lee C, Scherr HM, Wallingford JB: Shroom family proteins regulate gamma-tubulin distribution and microtubule architecture during epithelial cell shape change. Development 2007, 134:1431-1441.

doi:10.1186/2046-2530-1-13

Cite this article as: Thumberger et al: Ciliary and non-ciliary expression and function of PACRG during vertebrate development. Cilia 2012 1:13.

\section{Submit your next manuscript to BioMed Central and take full advantage of:}

- Convenient online submission

- Thorough peer review

- No space constraints or color figure charges

- Immediate publication on acceptance

- Inclusion in PubMed, CAS, Scopus and Google Scholar

- Research which is freely available for redistribution 